\title{
The Cellular Retinoic Acid Binding Protein 2 Promotes Survival of Malignant Peripheral Nerve Sheath Tumor Cells
}

\author{
Susan Fischer-Huchzermeyer, ${ }^{*}$ Anna Dombrowski, ${ }^{\top}$ Christian Hagel, ${ }^{\ddagger}$ Victor F. Mautner, ${ }^{\S}$ Jens Schittenhelm, ${ }^{\Uparrow}$ and Anja Harder ${ }^{* \|}$ \\ From the Institute of Neuropathology, * University Hospital Münster, Münster; the Clinics of Radiotherapy, ${ }^{\dagger}$ HELIOS Clinic Berlin-Buch, Berlin; the Institute \\ of Neuropathology $y^{\ddagger}$ and Clinics and Polyclinics of Neurology, ${ }^{\S}$ University Hospital Hamburg-Eppendorf, Hamburg; the Institute of Neuropathology, ${ }^{\llbracket}$ \\ University Hospital Tuebingen, Tuebingen; and the Institute of Pathology," Brandenburg Medical School, Brandenburg, Germany
}

\author{
Accepted for publication \\ February 27, 2017. \\ Address correspondence to \\ Anja Harder, M.D., Health Care \\ Center, Brandenburg Hospital, \\ Brandenburg Medical School, \\ Hochstr. 29, Brandenburg \\ 14770, Germany. E-mail: anja. \\ harder@mhb-fontane.de.
}

\begin{abstract}
Malignant peripheral nerve sheath tumors (MPNSTs) are aggressive neoplasms that commonly occur in patients with neurofibromatosis type 1 (NF1). Effective chemotherapy is not available. To characterize a therapeutic target for treatment, we investigated the role of cellular retinoic acid binding protein 2 (CRABP2) in MPNST in vitro. CRABP2 is a transcriptional co-activator of retinoic acid signaling. Although overexpression of CRABP2 is described in several cancers, it has not yet been studied in MPNSTs. We investigated CRABP2 expression in cultured Schwann cells and formalin-fixed, paraffin-embedded specimens of human peripheral nerve sheath tumors. A transient knockdown of CRABP2 was established in human NF1-associated MPNST cell lines (S462, T265, NSF1), and functional effects on viability, proliferation, apoptosis, and cytotoxicity were monitored. Finally, a 45-pathway reporter assay was performed in knockdown cells. Expression of CRABP2 was found in epithelium, fibroblasts, and tumor Schwann cells of skin, neurofibromas, and MPNSTs. In contrast, normal skin Schwann cells $\left(N F 1^{+/-}, N F 1^{-/-}\right)$ did not express CRABP2. In the absence of retinoic acid, MPNST cells depleted of CRABP2 had reduced viability and proliferation, induction of apoptosis and cytotoxicity, and up-regulation of the type 1 interferon pathway. These data suggest a retinoic acid-independent, non-tumor suppressor role of CRABP2 for the survival of MPNST cells in vitro. Targeting CRABP2 overexpression may represent a unique approach for the treatment of human MPNSTs. (Am J Pathol 2017, 187: 1623-1632; http://dx.doi.org/ 10.1016/j.ajpath.2017.02.021)
\end{abstract}

The cellular retinoic acid binding protein 2 (CRABP2) is an intracellular lipid-binding protein that binds retinoids and fatty acids, such as retinoic acid (RA). ${ }^{1}$ Expression is restricted to skin, uterus, ovary, choroid plexus, testis, and hematopoietic cells ${ }^{2-5}$ and can be up-regulated by alltrans retinoic acid (ATRA). The metabolite of vitamin A, ATRA, binds CRABP2 and acts as a co-activator of nuclear RA receptors and peroxisome proliferator-activated receptors. $^{6-8}$ The ligand-dependent transcription factors mediate transcriptional activation of ATRA-targeted pathways to regulate processes such as embryonic development, tissue remodeling, neuronal plasticity, lipid metabolism, and glucose homeostasis. ${ }^{7,9}$ Being a transcriptional co-activator, CRABP2 is involved in tissue differentiation and proliferation ${ }^{10}$ and in the regulation of axonal regeneration, neurite initiation, and neurite branching in mice. ${ }^{11}$

Abnormal regulation occurs in human disease and cancer. Thus, overexpression was detected in human tissue of leiomyoma, psoriatic skin, and bladder cancer and in cell lines of head and neck squamous cell carcinoma (SCC25) and breast cancer (MCF-7). ${ }^{12-16}$ Furthermore, CRABP2 serves as a biomarker in ovarian cancer and as a prognostic factor in Wilms tumors. ${ }^{17,18}$ High expression of CRABP2 correlates with improved prognosis in breast carcinoma. ${ }^{19}$ In medulloblastoma, CRABP2 expression correlates with

Supported by Deutsche Krebshilfe e.V. grant 109523 (A.H.) and Nothing Is Forever e.V. grant 604242 (A.H.).

Disclosures: None declared. 
ATRA response. ${ }^{20}$ Thus, CRABP2 is frequently overexpressed in disease and mediates ATRA sensitivity, implicating a potential therapeutic role.

In addition, CRABP2 possesses RA-independent functions first described in MCF-7 mammary carcinoma cells: in the absence of ATRA, overexpression of CRABP2 results in up-regulation of apoptotic protease activating factor 1 (APAF1) and triggers procaspase 7 and 9 cleavage. APAF1, a major protein of apoptosome formation, is not a direct target of ATRA. ${ }^{21}$ CRABP2 also posttranslationally regulates gene expression in carcinoma cells via mRNA stabilization of caspase 7 and other mRNA targets, in addition to transcriptional activation of ATRA-mediated pathways. ${ }^{22}$

So far, no data are available concerning involvement of CRABP2 in tumor development or progression of malignant peripheral nerve sheath tumors (MPNSTs). These soft-tissue sarcomas are associated with poor prognosis and 5-year survival rates as low as $26 \%$ to $60 \%$ attributable to early metastasis and recurrence. ${ }^{23-26}$ MPNSTs typically occur in patients with neurofibromatosis type 1 (NF1), arising from benign peripheral nerve sheath tumors, especially from large disease-specific plexiform neurofibromas. In NF1, biallelic inactivation of neurofibromin leads to overactivation of rat sarcoma followed by aberrant stimulation of downstream pathways. Molecular pathways that drive malignant transformation into MPNSTs are not fully understood. Additional genetic aberrations besides NFI inactivation are described.

In preliminary experiments, we observed CRABP2 expression in NF1-associated neurofibromas, the precursor lesion of MPNSTs in NF1, but not in normal Schwann cells. This finding suggests a role of CRABP2 for MPNST development. Furthermore, we found that CRABP2expressing MPNSTs respond to ATRA treatment in vitro. ${ }^{27}$ The present study focused on an RA-independent role of CRABP2 to elucidate its function in MPNST tumorigenesis.

\section{Material and Methods}

\section{Immunohistochemistry and Immunofluorescence of Formalin-Fixed, Paraffin-Embedded Material}

Human MPNST specimens from NF1 $(n=10)$ and non-NF1 patients $(n=6)$ and specimens of NF1-associated neurofibromas from 36 unrelated NF1 patients (17 cutaneous, 19 plexiform neurofibromas, World Health Organization grade I) were provided by German neuropathologic registries of University Hospital Muenster, University Hospital Hamburg-Eppendorf, HELIOS Clinic Emil von Behring Berlin, and University Hospital Tuebingen. Immunohistochemistry of full slides was performed using the Dako REAL Detection Kit (K5001) and AutostainerLink 48 (Dako Cytomation, Carpinteria, CA). Deparaffinized sections were incubated with primary anti-CRABP2 antibody (antibody 181255, 1:100; Abcam,
Cambridge, England). Immunofluorescence double labeling with antibodies against CD90, S100, and CRABP2 (kindly provided by $\mathrm{P}$. Chambon, Institute de Genetique, Strasbourg, France) combined with DAPI staining was performed in neurofibromas. For S100-CRABP2 double staining, sections were incubated with a mouse monoclonal anti-CRABP2 antibody (1:1000, overnight, $\left.4^{\circ} \mathrm{C}\right)$, followed by incubation with a Cy3-conjugated antibody (115-165003, 1:100; Dianova, Barcelona, Spain) for 1 hour. Afterward sections were incubated with anti-S100 antibody (Z0311; Dako Cytomation) for 2 hours, followed by incubation with a Cy2-conjugated antibody (111-165-003, 1:50; Dianova) and DAPI (32-804830; Vysis, Stuggart, Germany) for 1 hour. For CD90-CRABP2 double staining, deparaffinized sections were cooked in citrate buffer (10 minutes) followed by CRABP2 staining as described above. Afterward, sections were incubated with anti-CD90 antibody (Dia100, 1:50; Dianova) for 2 hours. Washed sections were incubated with Cy2-conjugated antibody and DAPI for 1 hour. Evaluation was performed using a fluorescence microscope (DFC490; Leica Microsystems, Wetzlar, Germany) and the program Leica Application Suite version 3.3 (Leica Microsystems).

\section{Cell Culture and RNA Interference by Transient Transfection of siRNA via HiPerFect}

Primary Schwann cells and primary fibroblasts derived from human NF1-derived neurofibromas were enriched and cultivated as described elsewhere. ${ }^{28}$ Primary cells were provided by V. Mautner (University of Hamburg, Hamburg, Germany) and procedures were approved by ethics committee at Ärztekammer Hamburg. Furthermore, NF1-associated MPNST cell lines S462, T265, and NSF1 were investigated. These cell lines were characterized for Schwann cell origin by S100 antibody staining permanently throughout our experiments. MPNST cell line S462 was established from an NF1-associated MPNST by S. Frahm in 2004 and provided by V. Mautner (University of Hamburg, Hamburg, Germany) being genetically characterized and followed by additional molecular analyses as published in detail elsewhere. ${ }^{29-31}$ T265 was also obtained from V. Mautner (University of Hamburg, Hamburg, Germany) and expressed approximately 900 abnormal genes. ${ }^{31}$ NF1-associated MPNST cell line NSF1 was kindly provided by D. Kaufmann (University Hospital Ulm, Ulm, Germany) from an NF1-associated MPNST and not characterized by extensive genetic analysis to our best knowledge. The $\mathrm{NF}^{-1-}$ status of these MPNSTs was not further monitored throughout the experiments. MPNST cell lines were cultured using Dulbecco's modified Eagle's medium complete containing $4.5 \mathrm{~g} / \mathrm{L}$ of glucose, $2 \mathrm{mmol} / \mathrm{L}$ of L-glutamine, $10 \%$ fetal bovine serum, $100 \mathrm{U} / \mathrm{mL}$ of penicillin/ streptomycin, and $1 \mathrm{mmol} / \mathrm{L}$ of sodium pyruvate in uncoated vessels. Fibroblast cell line 1BR3GN was purchased from the European collection of cell cultures and 
cultured in standard Dulbecco's modified Eagle's medium. The MCF-7 cell line derived from human breast carcinoma was kindly provided by Dr. V. Senner (University Hospital Muenster, Muenster, Germany) and cultured in standard Dulbecco's modified Eagle's mediumcomplete without sodium pyruvate. ${ }^{32}$ This cell line was used as a control for overexpression of CRABP2. Transient transfection of two specific siRNAs targeting CRABP2 mRNA [si-CRABP2-1 (AAUGGUCUGUGAGCAGAAG) and si-CRABP2-2 (AGAGGAAUGGCUGCAAGAG)] and an unspecific siRNA (si-scrambled, AllStars Negative Control siRNA; Qiagen, Hilden, Germany) as a negative control was performed via HiPerFect (Qiagen). Cells were seeded the day before transfection.

\section{In Vitro Assays}

Cell proliferation and migration of cells were analyzed using the xCELLigence DP system (Roche, Basel, Switzerland). Migration assays were performed in CIM plates and monitored for 24 hours. Cell proliferation was monitored continuously in E-plates for 120 hours. Viability of cells was determined using the MTT assay by incubation with 0.5 $\mathrm{g} / \mathrm{L}$ of MTT labeling solution or medium, followed by aspiration of medium, incubation with isopropanol (5 minutes), and measurement of absorbance performed at 560 $\mathrm{nm} / 750 \mathrm{~nm}$ in a GloMax-Multi+ Detection System (Promega, Madison, WI). The CytoTox-Glo Cytotoxicity Assay (Promega) and Caspase-Glo 3/7 Apoptosis Assay (Promega) were performed in white-walled 96-well plates. Luminescence signals were detected by Cyto Tox-Glo/ Caspase-Glo method (integration time each with 0.5 seconds) in a GloMax-Multi+ Detection System. Cells incubated with $10 \mu \mathrm{mol} / \mathrm{L}$ of staurosporine served as positive control.

\section{Luciferase Reporter Array}

A Cignal 45-Pathway Reporter Array (CCA-901L; Qiagen) was performed by transient co-transfection of $100 \mathrm{ng}$ of reporter-plasmid DNA and $15 \mathrm{nmol} / \mathrm{L}$ of siRNA using Lullaby reagent $(0.5 \mu \mathrm{L}$ per well; OZ Biosciences, San Diego, CA). Cells were seeded in RPMI 1640 medium complete without antibiotics, in white-walled 96-well plates, the day before transfection. Dual Luciferase Reporter Assay (Promega) was performed 48 to 72 hours after transfection via the Dual-Luciferase 2 injectors' method in a GloMaxMulti+ Detection System (specifications injector 1 and 2: volume, $100 \mu \mathrm{L}$; delay, 2 seconds; speed, $200 \mu \mathrm{L}$ per second; integration, 10 seconds).

\section{PCR}

Total RNA was isolated (Gene Elute Mammalian Total RNA Miniprep Kit; Sigma, St. Louis, MO), including DNase digestion with RNase-free DNase Set (Qiagen). cDNA was prepared with oligo (dT) primers and High Capacity cDNART Kit, including RNase inhibitor (Applied Biosystems, Foster City, CA). Quantitative real-time RT-PCR was performed using TaqMan Universal Master Mix (Applied Biosystems) in a StepOnePlus real-time PCR system (Applied Biosystems). Endogenous glyceraldehyde-3-phosphate dehydrogenase served as reference for calculating CRABP2 expression levels using the $\Delta \Delta C T$ method. Primer sequences are as follows: glyceraldehyde-3-phosphate dehydrogenase (forward: 5'-ACCCACTCCTCCACCTTTGAC-3', reverse: 5'-CATACCAGGAAATGAGCTTGACAA-3', probe: 5'CTGGCATTGCCCTCAACGACCA- $3^{\prime}$ ) and CRABP2 (catalog number 4331182; Gene Expression Assay; Thermo Fisher Scientific, Applied Biosystems, Waltham, MA).

\section{Western Blot Analysis}

To obtain whole cell protein extracts, cells were washed twice with cold phosphate-buffered saline and lyzed with radioimmunoprecipitation assay buffer containing protease inhibitors. Protein concentrations were determined using the Lowry method and the DCTM Protein Assay Kit (BioRad, Hercules, CA) according to the manufacturer's instructions. The absorbance at $750 \mathrm{~nm}$ was detected in a GloMaxMulti+ Detection System. Protein samples were separated by SDS-PAGE according to the Laemmli method at $120 \mathrm{~V}$ until the desired separation had been reached (1 to 1.5 hours). To determine molecular weight of detected protein bands, a prestained protein marker (Precision Plus ProteinTM Dual Color Standards, BioRad) was loaded. A tank-blotting chamber (Mini Trans Blot Cell, BioRad) was used to transfer proteins to a polyvinylidene difluoride membrane (Roche) at $250 \mathrm{~mA}$ for 45 minutes. After transfer, the polyvinylidene difluoride membrane was incubated with blocking solution followed by incubation with primary antibodies ( $\alpha$-CRABP2, 1:250, antibody 74365, Abcam; $\alpha$ - $\beta$-actin, 1:200,000, catalogue number 3700 , Cell Signaling, Danvers, MA) overnight at $4^{\circ} \mathrm{C}$. Afterward, the membrane was incubated in blocking solution, containing a horseradish peroxidase-conjugated secondary antibody ( $\alpha$-rabbit, 1:10,000, catalog number A2074, and $\alpha$-mouse, 1:10,000, catalogue number A3682, Sigma) at room temperature for 1 to 2 hours. Finally, the membrane was incubated with LuminataTM Forte Western Horseradish Peroxidase Substrate (Millipore, Billerica, MA) to quantify signals by the Chemi Doc MP Imaging system (BioRad) using Image Lab software version 4.1.0 (BioRad).

\section{Statistical Analysis}

Experiments were performed at least three times independently unless otherwise stated. $P<0.05$ was considered statistically significant. The $U$-test was performed to compare integration of the area under the curve between cells under CRABP2 knockdown and control cells. We did not perform statistical testing when the sample size was $\leq 3$. 
A
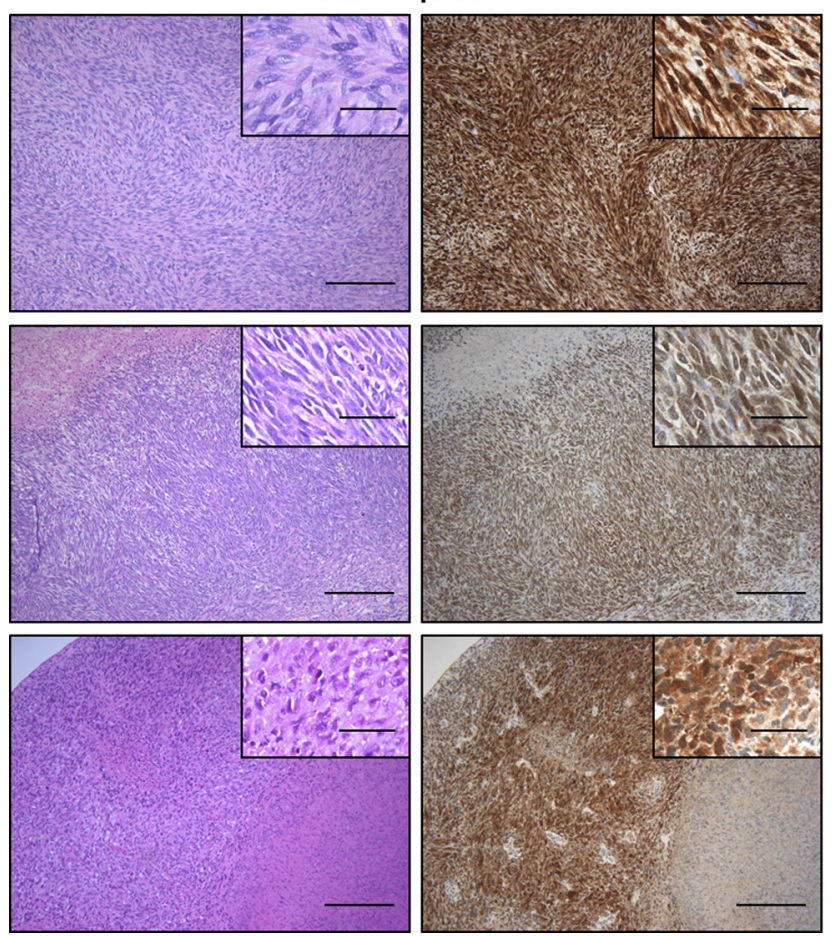

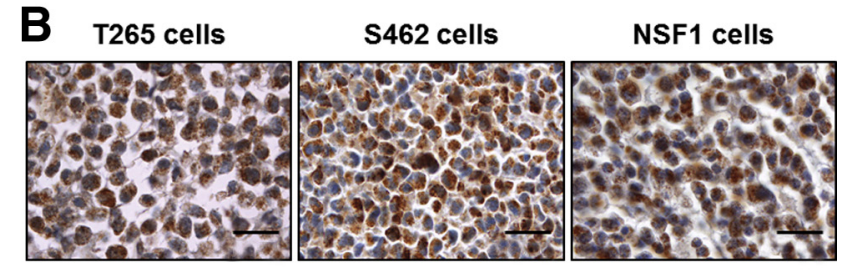

Figure 1 Expression of cellular retinoic acid binding protein 2 (CRABP2) in human malignant peripheral nerve sheath tumors (MPNSTs). Representative immunohistochemical pattern of CRABP2 in human neurofibromatosis type 1 (NF1)-associated MPNSTs, human non-NF1-associated MPNSTs, and human NF1-associated MPNST cell lines. A: Corresponding hematoxylin and eosin staining (left) and CRABP2 immunostaining (right) of three NF1-associated formalin-fixed, paraffin-embedded MPNST specimens revealing nuclear and cytoplasmic CRABP2 expression. B: CRABP2 immunostaining of MPNST cell lines T265, S462, and NSF1 revealing predominant cytoplasmic CRABP2 expression. $n=10$ (human NF1-associated MPNSTs); $n=6$ (human non-NF1-associated MPNSTs); and $n=3$ human NF1-associated MPNST cell lines. Scale bars: $250 \mu \mathrm{m}$ (A, main images); $50 \mu \mathrm{m}$ (A, insets); $500 \mu \mathrm{m}$ (B).

CRABP2 Depleted MPNST Cells Have Reduced Viability and Proliferation Accompanied by Increase of Apoptosis and Cytotoxicity in T265 Cells

CRABP2 Is Expressed in Epithelium, Fibroblasts, and Tumor Schwann Cells but Not in Normal $\left(N F 1^{+/+}\right)$ or Nontumor $\left(\mathrm{NF}^{+/-}\right)$Schwann Cells in Skin, Neurofibromas, and MPNSTs

Expression of $C R A B P 2$ in normal, benign, and malignant tumor Schwann cells, in epithelium, and in fibroblasts was determined. Human FFPE MPNST specimens $(n=16)$, NF1-associated MPNST cell lines (T265, S462, and NSF1), and NF1-associated cutaneous and plexiform FFPE neurofibromas $(n=36)$ were immunopositive for CRABP2, irrespective of NF1 background (Figure 1). Strong expression of CRABP2 was seen in epithelial cells of normal $\left(\mathrm{NFI}^{+/+}\right)$skin, including glands, and in epithelial cells of NF1 patients' skin $\left(N F 1^{+-}\right)$(Supplemental Figure S1). Fibroblasts of skin specimens or within neurofibromas expressed CRABP2. Cells had both nuclear and cytoplasmic staining. Only MPNST cells had a predominant cytoplasmic staining.

Expression in neurofibromas was studied in more detail. Double immunofluorescence labeling revealed complete co-expression of CD90 and CRABP2 in neurofibroma fibroblasts $\left(\mathrm{NFI}^{+-}\right)$, whereas a proportion of Schwann cells in neurofibromas did not express complete co-expression of S100 and CRABP2 (Supplemental Figures S2 and S3). Normal Schwann cells or NF1-associated Schwann cells of nerves $\left(\mathrm{NFI}^{+/-}\right)$within NF1-derived (tumor free) skin did not express CRABP2 immunohistochemically.
In the absence of RA, a transient knockdown of $C R A B P 2$ was established in the NF1-associated MPNST cell lines T265, S462, and NSF1 (Figure 2). Reduction of CRABP2 mRNA levels was found in all three MPNST cell lines, ranging from 0.04 - to 0.15 -fold in T265 cells, from 0.69 to 0.92 in S462 cells, and from 0.27 to 0.36 in NSF1 cells from day 1 to 5 after transfection: in T265 and S462 cells, viability was strongly reduced from day 3 by both siRNAs. Best knockdown efficiency was obtained in T265 cells (maximum fold-change reduction to 0.04 ) and verified by Western blot (Supplemental Figure S4). NSF1 cells also had a time-dependent reduction of viability after $C R A B P 2$ knockdown with si-CRABP2-2. Both siRNAs applied operated with similar efficiency in reducing CRABP2 mRNA levels.

The siRNA transfected cells were treated with ATRA and monitored (using the MTT assay for all three cell lines and the xCELLigence system to assess both effects on viability and proliferation in T265 cells). T265 cells were treated with $25 \mu \mathrm{M}$ ATRA from day 3 to 6 after transfection. $C R A B P 2$ expression was not detectable by real-time PCR and Western blot at day 3 to 6. ATRA treatment did not have an auxiliary effect on viability and proliferation in tumor cells with CRABP2 knockdown. No difference of viability (MTT, $n=4$ ) and proliferation (xCELLigence, $n=4$ ) was seen comparing T265 cells that lacked CRABP2 


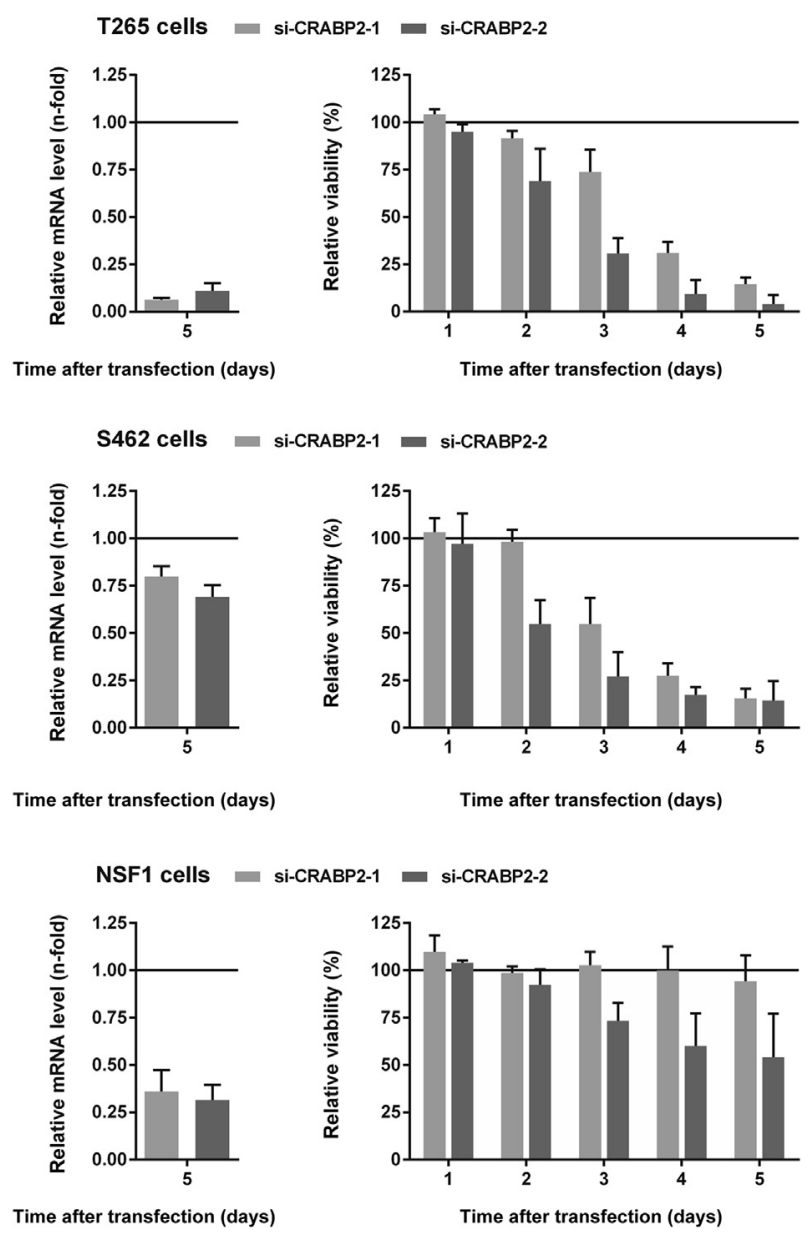

Figure 2 Viability in malignant peripheral nerve sheath tumor cell lines after cellular retinoic acid binding protein 2 (CRABP2) knockdown. Viability of cells (MTT assay) after transfection with specific siRNAs targeting CRABP2 mRNA [normalized to si-scrambled (100\%) (black line)] (right). Knockdown efficiency was determined via quantitative real-time RT-PCR (left). Bars indicate fold change of CRABP2 mRNA levels relative to nonspecific siRNA applied in control cells (black line, onefold). Data are expressed as means \pm SD.

under and without ATRA treatment (data not shown). These data suggest that the effects of ATRA are mediated by CRABP2; earlier findings indicate that CRABP2 expression decreases proliferation and migration of MPNST cells in vitro, similar to the effects of ATRA. ${ }^{27}$ Because $C R A B P 2$ knockdown was most effective in T265 cells, it was used for further investigation of proliferation (xCelligence system) and cytotoxic effects after transfection. Concordantly, proliferation of $C R A B P 2$-depleted cells was significantly lower compared with controls (Figure 3, A and B). Induced cytotoxic effects were seen after transfection, reaching a 2.2-fold to 2.4-fold induction (day 4) and a 1.7-fold to 2.1-fold induction (day 5) of relative number of dead cells after transfection with si-CRABP2-1 and si-CRABP2-2, respectively (Figure $3 C$ ). In addition, apoptosis was investigated by monitoring activation of caspase 3 and caspase 7 after transfection. CRABP2 knockdown in T265 cells induced apoptosis starting at day 2 with a 1.6-fold to 1.4-fold increase using si-CRABP2-1 and si-CRABP2-2, respectively (Figure 3D). Induction of caspase 3 and caspase 7 activities in CRABP2-depleted T265 cells was comparable to stauroporine treatment (positive control) and strongly induced compared with negative controls (si-scrambled) (Figure 3D).

CRABP2 Depletion Reduces Viability in MCF-7 Cells and Fibroblasts but Is Not Associated with Apoptosis and Cytotoxicity in Normal Fibroblasts

To elucidate whether the phenotype of $C R A B P 2$ knockdown is specific for MPNSTs, we assessed viability after $C R A B P 2$ knockdown in MCF-7 cells in primary cutaneous (cFib) and plexiform (pFib) NF1-associated neurofibroma-derived fibroblasts $\left(N F 1^{+--}\right)$and in normal fibroblasts (1BR3GN, $N F 1^{+/+}$). Knockdown efficiency was verified at mRNA level (Supplemental Figure S5). All cell types had reduced viability at days 1,3 , and 5 compared with control cells (si-scrambled), indicating stronger effects in normal and tumor-derived primary fibroblasts (strongly reduced viability) compared to MCF-7 cells (moderately reduced viability) (Figure 4).

Basal CRABP2 mRNA levels of different cell types were analyzed (Supplemental Figure S6). When normalized to CRABP2 mRNA levels of MCF-7 cells (100\%), normal primary fibroblasts had low levels (29\% to 33\%), and 1BR3GN cells had the lowest level (3\%). Expression of CRABP2 mRNA was variable in MPNST cells (9\% to $84 \%$ ), indicating high expression in T265 cells (84\%) being comparable to MCF-7 cells (Supplemental Figure S6).

Investigation of cytotoxic effects and apoptosis in 1BR3GN cells depleted of $C R A B P 2$ did not reveal increase of cytotoxicity or activation of caspase 3 and caspase 7 as described for T265 MPNST cells (Supplemental Figure S7).

\section{CRABP2 Depleted T265 MPNST Cells Involve CRABP2 in Interferon Regulation}

A 45-pathway reporter array comprising pathways, such as cellular stress response, stemness, and receptor tyrosine kinase, G-protein coupled receptor, and hormone signaling, was performed in CRABP2-depleted T265 cells by overexpression of reporter constructs and simultaneous gene silencing via siRNA (si-CRABP2-1). General pathway reporter activity independent of CRABP2 knockdown was investigated. A strong activity was found by the interferon-stimulated response element (MAPK/ ERK), AP1 (MAPK/JNK), and NF- $\kappa \mathrm{B}$ pathways (Figure 5A). Ten pathways (amino acid deprivation response element, androgen receptor, myocyte enhancer factor 2, interferon gamma activation sequence, retinoic acid response element, retinoid $\mathrm{x}$ receptor, sex determining region $\mathrm{Y}$-box 2, signal transducer and activator of transcription 3, T-cell factor/lymphoid enhancing factor, vitamin $\mathrm{D}$ receptor) had no activity (signals at background 
A

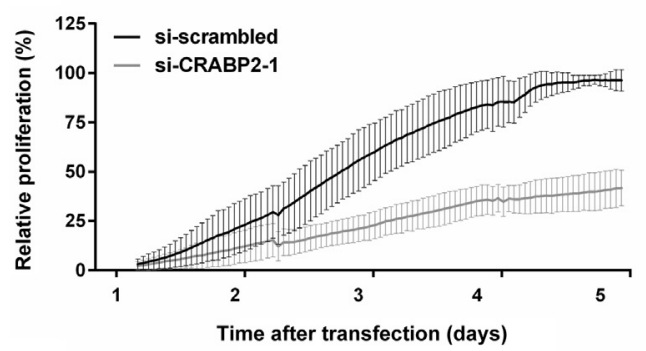

C

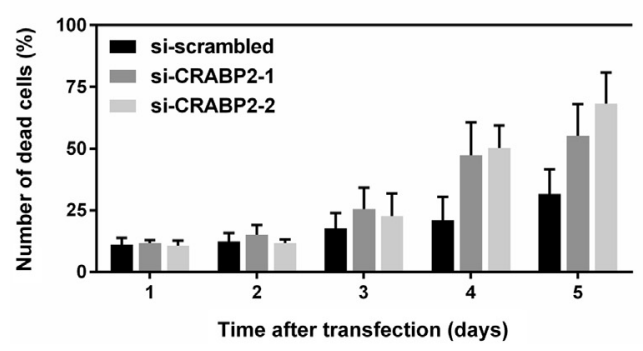

B

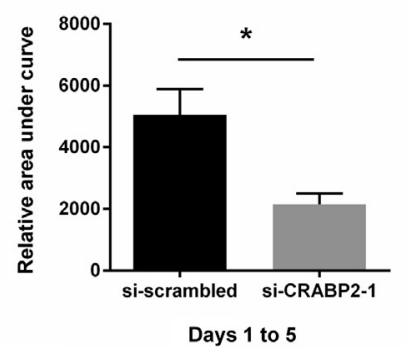

D

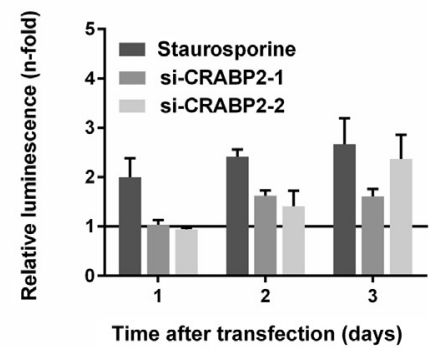

Figure 3 Effects of cellular retinoic acid binding protein 2 (CRABP2) knockdown on proliferation and induction of cytotoxicity and apoptosis in T265 cells. A: Reduced proliferation in T265 cells transfected with si-CRABP2-1 (gray line) or nonspecific si-scrambled RNA (black line) (xCELLigence system). B: Integration of the area under the curve (days 1 to 5 ) reveals significantly reduced proliferation of cells transfected with siCRABP2-1. C: Strong induction of cytotoxic effects (CytoTox-Glo assay, number of dead cells relative to all cells, black line, $100 \%$ ) in knockdown cells on days 4 and 5 after transfection with both siRNAs (gray bars) compared with cells transfected with nonspecific siRNA (black bars). D: Strong induction of luminescence signals (induction of apoptosis by activation of caspase 3 and 7 , Caspase-Glo $3 / 7$ assay) is observed in CRABP2 knockdown cells on days 2 and 3 after transfection (gray bars) compared with cells transfected with nonspecific siRNA (si-scrambled, black line, onefold). Cells treated with staurosporine (dark gray bars) served as positive control. Data are expressed as means \pm SD (B-D). ${ }^{*} P<0.05$ (U-test). levels) (Figure 5). To further analyze deregulation of pathways, luciferase signals of knockdown cells were normalized against control cells (Supplemental Table S1). Twelve pathway reporters had a $\log 2$ fold induction or reduction between 0.5 -fold and 1.0-fold. The interferonstimulated response element reporter had the highest ratio (1.5-fold), corresponding to an induction of reporter activity of 2.9-fold. The next most highly up-regulated reporter was interferon regulatory factor 1 , which was also involved in interferon regulation (Figure 6).

\section{Discussion}

We identified not only an RA-independent function of CRABP2 but a non-tumor suppressor role via maintaining cell survival of tumor cells, particularly MPNST cells. Although we found that other cells also respond to downregulation of CRABP2 with a decrease of viability, we did not detect induction of apoptosis or increased cytotoxicity in normal fibroblasts, indicating a cell-specific mode of action. Reduced viability of cells that did not undergo apoptosis after knockdown of $C R A B P 2$ can be explained by other mechanisms, such as cell cycle arrest, induction of senescence, or necrosis. Necrosis may be induced by cellular damages because of knockdown. Particular effects on cell cycle might be very informative but were not analyzed within this study and therefore warrant further investigation (eg, expression analysis of Ki-67 or $p 27$ ). Senescence could also be investigated by expression analysis of $\beta$-galactosidase in BRGN cells in further studies.

Expression of CRABP2 was seen in fibroblasts that had both nuclear and cytoplasmic staining, whereas MPNST cells had a predominant cytoplasmic staining in our study. This finding could probably be associated with cell type-specific up- and down-regulation of proteins that regulate nuclear protein import, such as human antigen R (HUR). ${ }^{33}$

RA-independent functions of CRABP2 were first described in MCF-7 breast carcinoma cells overexpressing CRABP $2,{ }^{21}$ leading to up-regulation of APAF1 expression and triggering procaspase 7 and 9 cleavage. More precisely,

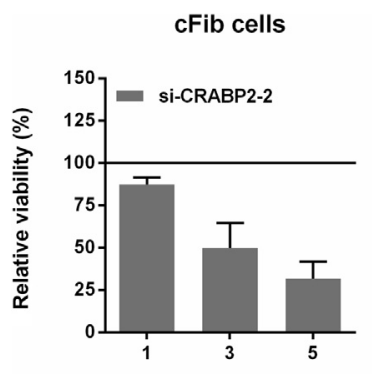

Time after transfection (days)

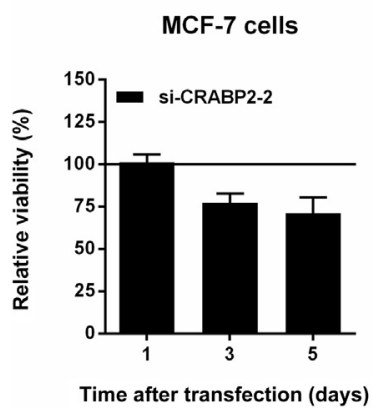

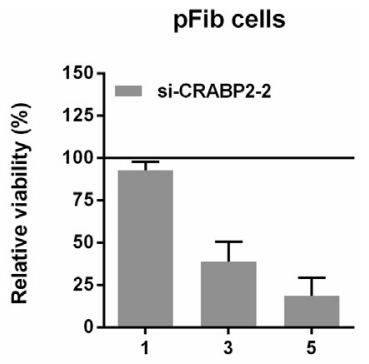

Time after transfection (days)

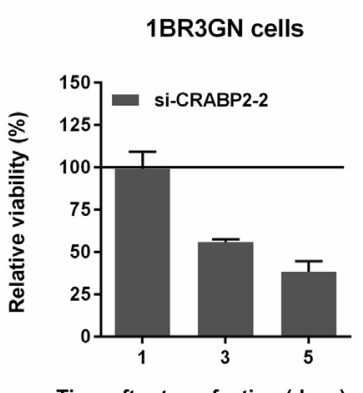

Time after transfection (days)
Figure 4 Relative viability after cellular retinoic acid binding protein 2 (CRABP2) knockdown in different tumor cells and 1BR3GN cells. Viability (MTT assay) under CRABP2 knockdown in MCF-7 cells, fibroblasts derived from cutaneous fibroblast (cFib) and plexiform fibroblast (pFib) NF1associated neurofibromas, and $1 \mathrm{BR} 3 \mathrm{GN}$ cells. CRABP2 knockdown was conducted using si-CRABP2-2 (gray bars) and si-scrambled (black line, $100 \%)$ as control. Data are expressed as means \pm SD. 
A

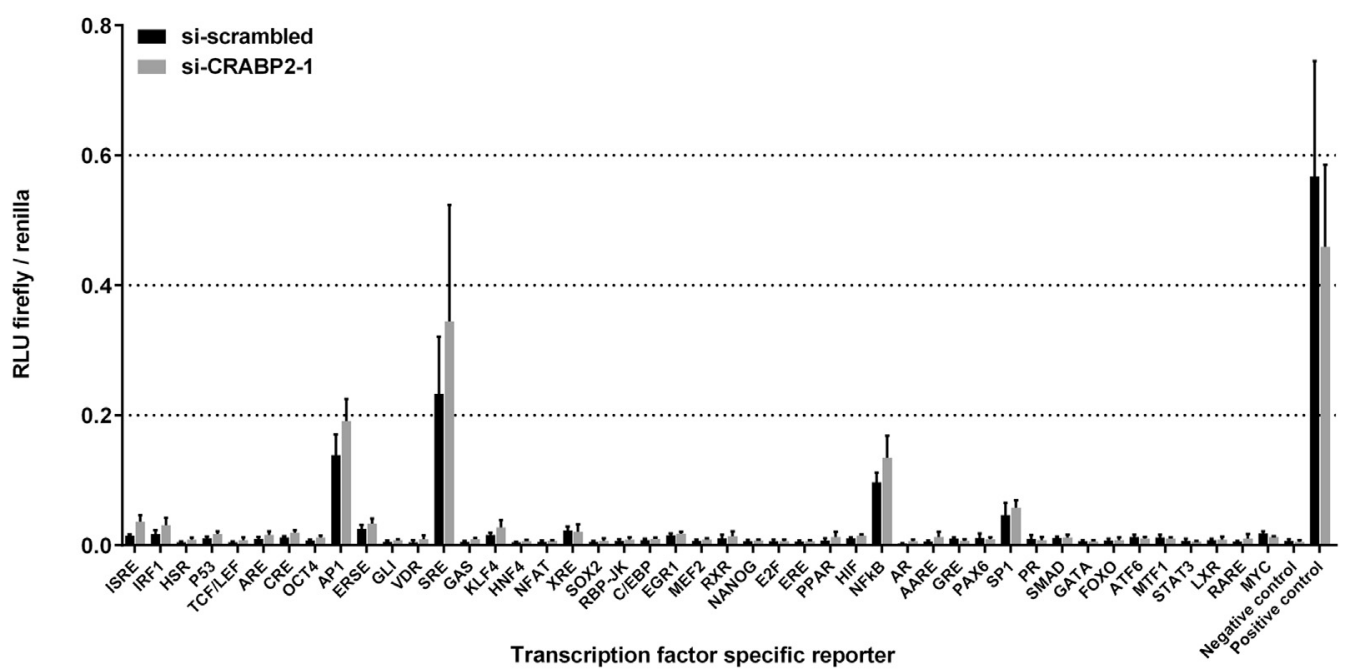

B

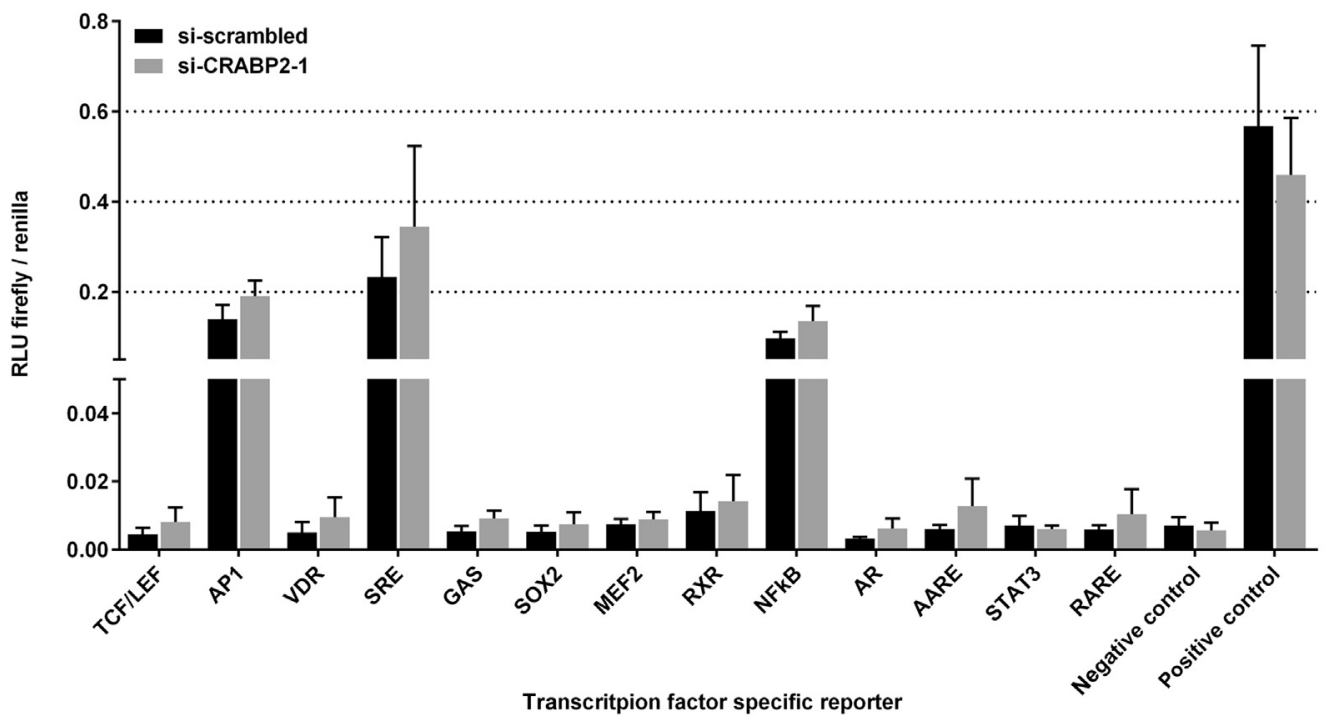

Figure 5 A 45-pathway reporter array in T265 MPNST cells. A: Relative luminescence units (RLU) of firefly/renilla luciferase signals are shown for each pathway reporter in malignant peripheral nerve sheath tumor T265 knockdown cells (si-CRABP2-1, gray bars) and control cells (si-scrambled, black bars), as well as positive and negative controls. B: Excerpt of A, revealing three pathway reporters that were highly active (AP1, interferon-stimulated response element, and NF- $\mathrm{B}$ ) and 10 pathway reporters that were inactive, with luciferase signals at background levels, in knockdown cells and control cells. Data are expressed as means \pm SEM.

CRABP2 was described to be involved in posttranslational gene regulation via interaction with HUR that is known to bind $\mathrm{AU}$ rich elements of $3^{\prime}$ untranslated region of target mRNAs. ${ }^{22}$ Binding of CRABP2 to HUR increased affinity of HUR to AU rich elements of APAF1 mRNA, resulting in transcript stabilization. Cell proliferation was suppressed by stabilization of antiproliferative HUR target transcripts. Those studies suggested that CRABP2 inhibits proliferation of carcinoma cells via transcriptional activation of ATRAmediated signaling pathways and posttranslational mRNA stabilization of caspase 7 and other mRNA targets. ${ }^{22}$ Our study found that CRABP2 similarly acts independent of RA in MPNSTs while maintaining cell survival attributable to down-regulation of apoptosis and cytotoxicity as shown in T265 MPNST cells. Principally, enhancement of cytotoxicity and apoptosis attributable to $C R A B P 2$ knockdown should necessarily be found in further studies in more cell lines and cancers. Knockdown of $C R A B P 2$ in MPNSTs that resulted in reduced viability and proliferation may point to a therapeutic approach via direct targeting CRABP2 degradation in MPNSTs.

Normally, CRABP2 is expressed in epithelium, ovary, uterus, prostate, testicle, kidney, eye, and embryonic tissue but not in Schwann cells. ${ }^{34-39}$ Consistently, we did not find CRABP2 expression in peripheral nerves. However, Schwann cells of NF1-associated neurofibromas expressed CRABP2, although only in a subpopulation of cells. This finding implicates a transformation-induced up-regulation of 


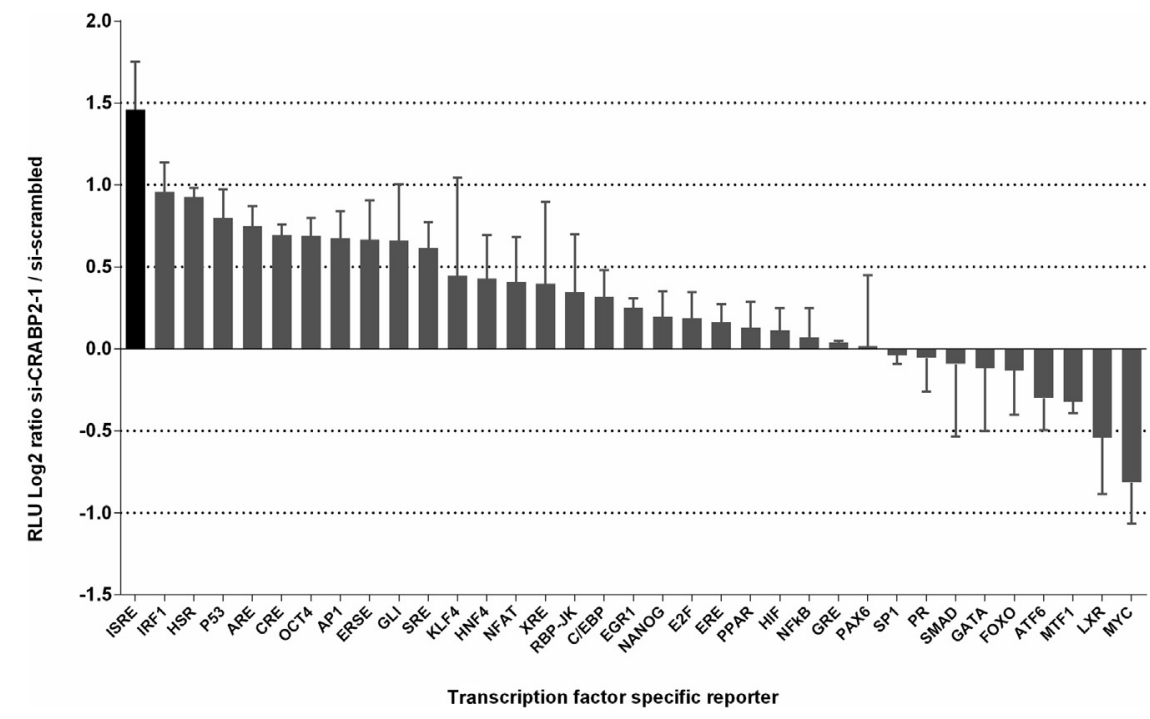

Figure 6 Regulation of pathway reporters in cellular retinoic acid binding protein 2 (CRABP2)depleted T265 cells. Pathway reporters are listed for decreasing values of log2 ratio fold-changes (log2 ratio of si-CRABP2-1/si-scrambled). Reporters above a ratio of zero are up-regulated in knockdown cells, whereas reporters below a ratio of zero are downregulated in knockdown cells compared with control cells. The interferon-stimulated response element reporter reveals the highest induction with a log2 ratio of 1.5 0.3-fold in T265 cells. Data are expressed as means \pm SEM.
CRABP2 in tumor Schwann cells of benign Schwann cell-derived tumors and in malignant tumor Schwann cells as described for MPNSTs. Although CRABP2 is involved in neurite initiation and branching during axonal regeneration and is also up-regulated because of nerve injury, we argue that CRABP2 may be differentially regulated in Schwann cells. ${ }^{11,40,41}$ Similarities between gene expression in wound healing and tumor development may be associated with CRABP2 up-regulation in benign neurofibromas. Interestingly, in fibroblasts of scars, expression of genes such as $N F 1$ is up-regulated, and $N F 1^{+-}$mice have disturbed wound healing accompanied by collagen deposition and fibroblast hyperplasia. The latter is similarly seen in neurofibroma formation. Furthermore, overexpression of $C R A B P 2$ in neuroblastoma cell induced cell motility in wound healing and migration assays. ${ }^{42}$ We therefore hypothesize that up-regulation of CRABP2 in Schwann cells of NF1 mutation-associated neurofibromas underlies processes that are identical to wound healing, including axonal regeneration and increased cell motility, at least in cutaneous neurofibroma-derived Schwann cells. This finding should prompt further detailed studies. In our study, a differential $C R A B P 2$ expression in cutaneous versus plexiform neurofibromas was not obvious. Cutaneous and plexiform neurofibromas have an entirely different biological behavior regarding malignant transformation. Cutaneous neurofibromas derive from $\mathrm{NF}^{-1-}$ skin-derived progenitor cells and never turn malignant, whereas plexiform neurofibromas develop from $\mathrm{NF}^{-1-}$ embryonic stem cells and are at risk for malignant change. From our data, we conclude that CRABP2 is probably not involved in early tumor initiation as it was found for Runx 1 but may be involved in later processes during transformation, which is also associated with $S U Z 12, C D K N 2 A$, and $E E D$ alterations and $K D M 2 B$ mutations. $^{43,44}$

Finally, we identified a so far unknown target of $C R A B P 2$ down-regulation in MPNST, the type 1 interferon pathway.
Depletion of $C R A B P 2$ up-regulates this pathway, therefore indicating that this immune-stimulating, antiviral, and antitumorigenic pathway is suppressed in MPNST overexpressing CRABP2. Interestingly, a recent study identified higher serum concentrations of interferon- $\gamma$ in NF1 patients compared with healthy controls. ${ }^{45}$ So far, alterations of the type $1(\alpha)$ interferon pathway have not yet been investigated in MPNSTs. A case study of a patient with a malignant triton tumor reported a long survival of $>3$ years under combined therapy of isotretinoin and interferon- $\alpha .{ }^{46}$ Those single efforts combined with our in vitro analyses may uncover a therapeutic role of the interferon- $\alpha$ pathway in MPNSTs.

summarize, we found expression of CRABP2 in human tumor Schwann cells and that loss of CRABP2 in MPNSTs reduces viability and proliferation but induces apoptosis, cytotoxicity, and interferon- $\alpha$ signaling. This study suggests that CRABP2 may be mandatory for cell survival. To establish novel options for MPNST treatment, these experiments should be verified by in vivo experiments, such as MPNST xenograft nude mouse models. To enforce the relevance of CRABP2 as therapeutic target, several further experiments can be imagined. For example, injection of lentiviral particles expressing CRABP2-specific shRNAs into xenografts or systemic treatment of mice with MPNST cell-specific aptamers being coupled to siRNA specific for CRABP2 or to hybrid molecules that induce proteasomal CRABP2 degradation may be conceivable. Targeting malignant tumors with tools that target degradation of CRABP2 only in tumor cells could circumvent potential resistance toward chemotherapeutics. Regulation of the interferon- $\alpha$ pathway may represent another option for in vivo experimental testing.

\section{Acknowledgments}

We thank Theresa Weiland and Eric Tietz for technical support; Hans-Joachim Galla and Werner Paulus 
(University of Münster) for encouraging this research; and Pierre Chambon (Institute de Genetique, Strasbourg, France), Dieter Kaufmann (University Hospital Ulm, Germany), and Dr. Volker Senner (University Hospital Muenster, Germany) for CD90, S100, and CRABP2 antibodies, NSF1 cell line, and human breast carcinoma-derived MCF7 cell line, respectively.

\section{Supplemental Data}

Supplemental material for this article can be found at http://dx.doi.org/10.1016/j.ajpath.2017.02.021.

\section{References}

1. Vaezeslami S, Mathes E, Vasileiou C, Borhan B, Geiger JH: The structure of Apo-wild-type cellular retinoic acid binding protein II at 1.4 A and its relationship to ligand binding and nuclear translocation. J Mol Biol 2006, 363:687-701

2. Wardlaw SA, Bucco RA, Zheng WL, Ong DE: Variable expression of cellular retinol- and cellular retinoic acid-binding proteins in the rat uterus and ovary during the estrous cycle. Biol Reprod 1997, 56:125-132

3. Zheng WL, Bucco RA, Schmitt MC, Wardlaw SA, Ong DE: Localization of cellular retinoic acid-binding protein (CRABP) II and CRABP in developing rat testis. Endocrinology 1996, 137:5028-5035

4. Kreutz M, Fritsche J, Andreesen R, Krause SW: Regulation of cellular retinoic acid binding protein (CRABP II) during human monocyte differentiation in vitro. Biochem Biophys Res Commun 1998, 248: 830-834

5. Yamamoto M, Drager UC, Ong DE, McCaffery P: Retinoid-binding proteins in the cerebellum and choroid plexus and their relationship to regionalized retinoic acid synthesis and degradation. Eur J Biochem 1998, 257:344-350

6. Chambon P: A decade of molecular biology of retinoic acid receptors FASEB J 1996, 10:940-954

7. Michalik L, Wahli W: Guiding ligands to nuclear receptors. Cell 2007 , 129:649-651

8. Schroeder F, Petrescu AD, Huang H, Atshaves BP, McIntosh AL, Martin GG, Hostetler HA, Vespa A, Landrock D, Landrock KK, Payne HR, Kier AB: Role of fatty acid binding proteins and long chain fatty acids in modulating nuclear receptors and gene transcription. Lipids 2008, 43:1-17

9. Desvergne B, Michalik L, Wahli W: Transcriptional regulation of metabolism. Physiol Rev 2006, 86:465-514

10. Eller MS, Muz P, Gilchrest BA: Regulation of CRABP II mRNA expression in human keratinocytes. Exp Dermatol 1995, 4:97-103

11. Dieplinger B, Schiefermeier N, Juchum-Pasquazzo M, Gstir R, Huber LA, Klimaschewski L, Vietor I: The transcriptional corepressor TPA-inducible sequence 7 regulates adult axon growth through cellular retinoic acid binding protein II expression. Eur J Neurosci 2007, 26: $3358-3367$

12. Tsibris JC, Segars J, Coppola D, Mane S, Wilbanks GD, O'Brien WF, Spellacy WN: Insights from gene arrays on the development and growth regulation of uterine leiomyomata. Fertil Steril 2002, 78: $114-121$

13. Siegenthaler G, Tomatis I, Didierjean L, Jaconi S, Saurat JH: Overexpression of cellular retinoic acid-binding protein type II (CRABP-II) and down-regulation of CRABP-I in psoriatic skin. Dermatology 1992, $185: 251-256$

14. Jin BY, Fu GH, Jiang X, Pan H, Zhou DK, Wei XY, Zhou L, Chung L, Zheng SS: CRABP2 and FABP5 identified by 2D DIGE profiling are upregulated in human bladder cancer. Chin Med J (Engl) 2013, 126: $3787-3789$
15. Hathout Y, Riordan K, Gehrmann M, Fenselau C: Differential protein expression in the cytosol fraction of an MCF-7 breast cancer cell line selected for resistance toward melphalan. J Proteome Res 2002, 1: 435-442

16. Vo HP, Crowe DL: Transcriptional regulation of retinoic acid responsive genes by cellular retinoic acid binding protein-II modulates RA mediated tumor cell proliferation and invasion. Anticancer Res 1998, 18:217-224

17. Toyama A, Suzuki A, Shimada T, Aoki C, Aoki Y, Umino Y, Nakamura Y, Aoki D, Sato TA: Proteomic characterization of ovarian cancers identifying annexin-A4, phosphoserine aminotransferase, cellular retinoic acid-binding protein 2, and serpin B5 as histologyspecific biomarkers. Cancer Sci 2012, 103:747-755

18. Gupta A, Kessler P, Rawwas J, Williams BR: Regulation of CRABP-II expression by $\mathrm{MycN}$ in Wilms tumor. Exp Cell Res 2008, 314: $3663-3668$

19. Bertucci F, Houlgatte R, Benziane A, Granjeaud S, Adelaide J, Tagett R, Loriod B, Jacquemier J, Viens P, Jordan B, Birnbaum D, Nguyen C: Gene expression profiling of primary breast carcinomas using arrays of candidate genes. Hum Mol Genet 2000, 9:2981-2991

20. Fu YS, Wang Q, Ma JX, Yang XH, Wu ML, Zhang KL, Kong QY, Chen XY, Sun Y, Chen NN, Shu XH, Li H, Liu J: CRABP-II methylation: a critical determinant of retinoic acid resistance of medulloblastoma cells. Mol Oncol 2012, 6:48-61

21. Donato LJ, Noy N: Suppression of mammary carcinoma growth by retinoic acid: proapoptotic genes are targets for retinoic acid receptor and cellular retinoic acid-binding protein II signaling. Cancer Res 2005, 65:8193-8199

22. Vreeland AC, Yu S, Levi L, de Barros Rossetto D, Noy N: Transcript stabilization by the RNA-binding protein $\mathrm{HuR}$ is regulated by cellular retinoic acid-binding protein 2. Mol Cell Biol 2014, 34: $2135-2146$

23. Dozois EJ, Wall JC, Spinner RJ, Jacofsky DJ, Yaszemski MJ, Sim FH, Moran SL, Cima RR, Larson DR, Haddock MG, Okuno SH, Larson DW: Neurogenic tumors of the pelvis: clinicopathologic features and surgical outcomes using a multidisciplinary team. Ann Surg Oncol 2009, 16:1010-1016

24. Goertz O, Langer S, Uthoff D, Ring A, Stricker I, Tannapfel A, Steinau HU: Diagnosis, treatment and survival of 65 patients with malignant peripheral nerve sheath tumors. Anticancer Res 2014, 34: $777-783$

25. LaFemina J, Qin LX, Moraco NH, Antonescu CR, Fields RC, Crago AM, Brennan MF, Singer S: Oncologic outcomes of sporadic, neurofibromatosis-associated, and radiation-induced malignant peripheral nerve sheath tumors. Ann Surg Oncol 2013, 20:66-72

26. Stucky CC, Johnson KN, Gray RJ, Pockaj BA, Ocal IT, Rose PS, Wasif N: Malignant peripheral nerve sheath tumors (MPNST): the Mayo Clinic experience. Ann Surg Oncol 2012, 19:878-885

27. Fischer S, Dombrowski A, Wilke G, Mautner VF, Friedrich RE, Heppner F, Harder A: Phenotypes of NF1 associated peripheral nerve sheath tumors are associated with modified retinoic acid signaling mediated by differential CRABPII expression. 57th Annual Meeting of the German Society for Neuropathology and Neuroanatomy (DGNN). Dusseldorf, Germany: German Medical Science GMS Publishing House, 2012.

28. Wallace MR, Rasmussen SA, Lim IT, Gray BA, Zori RT, Muir D: Culture of cytogenetically abnormal Schwann cells from benign and malignant NF1 tumors. Genes Chromosomes Cancer 2000, 27: $117-123$

29. Frahm S, Mautner VF, Brems H, Legius E, Debiec-Rychter M, Friedrich RE, Knofel WT, Peiper M, Kluwe L: Genetic and phenotypic characterization of tumor cells derived from malignant peripheral nerve sheath tumors of neurofibromatosis type 1 patients. Neurobiol Dis 2004, 16:85-91

30. Holtkamp N, Okuducu AF, Mucha J, Afanasieva A, Hartmann C, Atallah I, Estevez-Schwarz L, Mawrin C, Friedrich RE, Mautner VF, von Deimling A: Mutation and expression of PDGFRA and KIT in 
malignant peripheral nerve sheath tumors, and its implications for imatinib sensitivity. Carcinogenesis 2006, 27:664-671

31. Lee PR, Cohen JE, Tendi EA, Farrer R, DE Vries GH, Becker KG, Fields RD: Transcriptional profiling in an MPNST-derived cell line and normal human Schwann cells. Neuron Glia Biol 2004, 1:135-147

32. Soule HD, Vazguez J, Long A, Albert S, Brennan M: A human cell line from a pleural effusion derived from a breast carcinoma. J Natl Cancer Inst 1973, 51:1409-1416

33. Zhang W, Vreeland AC, Noy N: RNA-binding protein HuR regulates nuclear import of protein. J Cell Sci 2016, 129:4025-4033

34. Donovan M, Olofsson B, Gustafson AL, Dencker L, Eriksson U: The cellular retinoic acid binding proteins. J Steroid Biochem Mol Biol 1995, 53:459-465

35. Torma H, Lontz W, Liu W, Rollman O, Vahlquist A: Expression of cytosolic retinoid-binding protein genes in human skin biopsies and cultured keratinocytes and fibroblasts. Br J Dermatol 1994, 131:243-249

36. Si SP, Tsou HC, Lee X, Peacocke M: Effect of cellular senescence and retinoic acid on the expression of cellular retinoic acid binding proteins in skin fibroblasts. Exp Cell Res 1995, 219:243-248

37. Elder JT, Astrom A, Pettersson U, Tavakkol A, Griffiths CE, Krust A, Kastner P, Chambon P, Voorhees JJ: Differential regulation of retinoic acid receptors and binding proteins in human skin. J Invest Dermatol 1992, 98:673-679

38. Dong D, Ruuska SE, Levinthal DJ, Noy N: Distinct roles for cellular retinoic acid-binding proteins I and II in regulating signaling by retinoic acid. J Biol Chem 1999, 274:23695-23698
39. Nezzar H, Chiambaretta F, Marceau G, Blanchon L, Faye B, Dechelotte P, Rigal D, Sapin V: Molecular and metabolic retinoid pathways in the human ocular surface. Mol Vis 2007, 13:1641-1650

40. Zhelyaznik N, Schrage K, McCaffery P, Mey J: Activation of retinoic acid signalling after sciatic nerve injury: up-regulation of cellular retinoid binding proteins. Eur J Neurosci 2003, 18:1033-1040

41. Carroll SL, Ratner N: How does the Schwann cell lineage form tumors in NF1? Glia 2008, 56:1590-1605

42. Gupta A, Williams BR, Hanash SM, Rawwas J: Cellular retinoic acidbinding protein II is a direct transcriptional target of $\mathrm{MycN}$ in neuroblastoma. Cancer Res 2006, 66:8100-8108

43. Li H, Zhao X, Yan X, Jessen WJ, Kim MO, Dombi E, Liu PP Huang G, Wu J: Runx1 contributes to neurofibromatosis type 1 neurofibroma formation. Oncogene 2016, 35:1468-1474

44. Sohier P, Luscan A, Lloyd A, Ashelford K, Laurendeau I, BriandSuleau A, Vidaud D, Ortonne N, Pasmant E, Upadhyaya M: Confirmation of mutation landscape of NF1-associated malignant peripheral nerve sheath tumors. Genes Chromosomes Cancer 2017 , $56: 421-426$

45. Park SJ, Sawitzki B, Kluwe L, Mautner VF, Holtkamp N, Kurtz A: Serum biomarkers for neurofibromatosis type 1 and early detection of malignant peripheral nerve-sheath tumors. BMC Med 2013, 11:109

46. Kostler WJ, Amann G, Grunt TW, Singer CF, Schneider SM, Brodowicz T, Tomek S, Zielinski CC: Recurrent malignant Triton tumour: first report on a long time survivor. Oncol Rep 2003, 10: $533-535$ 